\title{
Risk assessment: information for qualitative estimation of probability and severity in high risk work context
}

\author{
A.A. Ribeiro \\ Instituto Superior de Línguas e Administração de Leiria, Leiria, Portugal
}

J.P.C. Fernandes Thomaz

Centro de Estudos de Gestão do Instituto Superior Técnico, Universidade de Lisboa, Lisboa, Portugal

Instituto Superior de Línguas e Administração de Leiria, Leiria, Portugal

R.A.C. Veiga

Instituto Superior de Línguas e Administração de Santarém, Santarém, Portugal

The process of risk management is a structured study which consists of three basic steps; risk analysis, risk assessment and risk control. Risk analysis is the first step of the risk assessment process and comprises the identification of all hazards present in the activities, and the estimation of probability and severity according to the method of risk assessment chosen.

This article intends to present the results of a study conducted in order to identify the information considered essential for a qualitative estimation of probability and severity to facilitate the technician's job during risk assessment when they use the semi-quantitative risk assessment methods (SqtRAM).

\section{INTRODUCTION}

The occurrence of accidents allowed in most cases to establish a causal link leading to the notion of risk and the understanding of the need for its management. In today's legal framework special emphasis is given to duties and responsibilities of the participants involved in risk assessment (RA) as a crucial element of the prevention process, providing knowledge of the existence of hazards (naturemagnitude) and contributing with information for risk control and development of preventive actions.

Although the freedom to select the methodology, the method chosen must be able to discriminate the different levels of risk (LR) in each situation, taking into account the parameters of the probability $(\mathrm{P})$ and severity (S), and its weight to achieve this goal.

The semi-quantitative risk assessment methods (SqtRAM) are used in most cases because they are generalist methods, easy to apply and a tool available to carry out the obligations imposed by law, in opposition to quantitative methods, which are more complex and therefore, involve higher costs.

\subsection{Definition of the research issues}

In the definition of the objectives, we attempted to find answers for the matter of qualitative risk estimation. To achieve the objectives of the study the following central research question was drawn up:

CRQ - Which are the markers of essential information for qualitative estimation of the variables Probability and Severity when using SqtRAM?

This question guided to the following derivative questions (DQ) and hypotheses (H):
DQ1 - Is the risk level obtained with SqtRAM significantly influenced by the information chosen for estimation of Probability and Severity?

$\mathrm{H} 0$ - There are significant differences in risk level obtained when we use distinct information for estimation.

H1 - There are no significant differences in risk level obtained when we use distinct information for estimation.

DQ2 - Does the type of assessed risk influence the choice of information for qualitative estimation of Probability and Severity, in SqtRAM?

HO - The type of assessed risk influences the choice of information for estimation.

H1 - Type of assessed risk does not influence the choice of information for estimation.

\subsection{Estimation of probability and severity variables}

The process of risk assessment and management consists in a structured study of all aspects inherent to the work and is composed of risk analysis, risk assessment and risk control. Risk analysis is performed to identify all hazards in the organizations activities and the estimation of probability and severity according to the method chosen $-\mathrm{R}=\mathrm{P} \times \mathrm{S}$ (Nunes 2010 \& Freitas 2008).

Estimating the risk, means measuring its magnitude, which is the product of the probability of the damage (estimated probability) by the severity (estimate of the damage), as objectively as possible. The risk estimation, should consider the systems and control measures already implemented, as well as information that may influence the results (Lluna 2003 \& Romero 2004). 
It is extremely helpful to understand the estimation of these two variables to illustrate the preventive measures that have the main objective of reducing the likelihood of exposure (actions taken before exposure to the risk) and collective or individual protective measures aiming to reduce the consequences due to exposure (actions required after exposure to reduce risk impact).

Lluna (2003) refers that the estimation highlights the sensitivity of the results due to its influence on preventive measures, as an important aspect when deciding on the measures to be implemented.

\subsection{Estimation of the probability and severity}

Estimating means forming an opinion based on an approximate judgment (based on predefined reference parameters). The question is, estimating what?

The probability is evaluated by different approaches. It is related to the possible occurrence of damage loss or consequence due to the conditions of use, exposure or interaction with the material component of work that presents a danger. In particular, when the working conditions determine the type of worker exposure, considering the number of times and / or duration of exposure to risk.

Estimating the severity, according to the approach taken, is interpreted as the consequences caused by the occurrence of exposure to risk (the potential severity of the damage).

The exposure to a particular occupational risk in the workplace which can generate different consequences, estimation of severity should meet the following points: (i) the damage perceived as more serious for the person or group with the likelihood of exposure, (ii) attend the exposure of workers considered most vulnerable to risk (pregnant women, children, disabled, elderly, illiterate) and (iii) take into account cases where the risk being assessed has a high level of risk resulting from the combination with the variable probability.

\section{RESEARCH METHODOLOGY}

This study used a qualitative research approach, with the main objective of identifying the information needed for qualitative estimation of probability and severity in risk analysis, the first step of risk management.

To reach this goal, the content analysis of data (interviews) was done through the interpretation of the concepts expressed in a qualitative and cognitive analysis following two complementary techniques:

(i) Triangulation technique as a way to avoid individual analysis on the basis of personal opinion of the researcher, so that appealed to three independent analysts (Northcutt \& McCoy 2004);

(ii) Cognitive mapping technique for aggregation of concepts and their clarification, according to the
Strategic Options Development and Analysis approach (Eden \& Ackermann 1998).

This method was selected because it allows us to describe and interpret qualitative estimation of risk, without any control or interference in; existing work conditions, instruments for data collection and data analysis.

\subsection{Steps of the research}

The research took place in five key steps:

Step 1 - Definition of indicators that characterize the utilization of explosives for rock dismantling in extractive industry (workplace case study);

Step 2 - Observation, systematically directed to the collection of data in the workplace;

Step 3 - Definition of the criteria for selection the sample of interviewees;

Step 4 - Execution of interviews to the sample and presentation of workplace/activity observed;

Step 5 - Transcription of interviews and data analysis with qualitative analysis techniques;

Step 6 - Analysis of estimation information identified in "counterpoint" to the variables of three semi-quantitative risk assessment methods.

\subsection{Data collection techniques}

In data collection were applied the following two instruments:

(i) A planned and participated observation of the workplace where explosives are used for rock dismantling in a quarry (high risk job) - Step 2;

(ii) Individual, semi-structured interviews carried out with risk assessment teachers and risk managers of medium and large companies - Step 4.

\subsection{Observation of workplace - case study}

The observation of the workplace went through the following phases: definition of objectives, site and work selection, identification of indicators to characterize the workplace and implementation of observation plan (timing of visits and data collection).

During the observation of rock dismantling with explosives the following indicators were assessed: (i) organization, processes and work procedures, (ii) technical, technological and products used, (iii) physical environment and (iv) the human factors (step 1 and 2).

\subsection{Individual semi-structured interview}

The selection procedure of respondents was made by a non-probabilistic rational or typical cases sampling (Fortin 2006) -Step 3.

This option was due to two key factors, the specific subject of study, and the difficulty of defining and accessing the entire population of teachers of risk assessment and risk managers of medium and large companies. To form the sample the following criteria and purpose were taken into account: 
(i) The technical and scientific character of the data and avoid the saturation of information (data repetition);

(ii) Be risk assessment teachers or responsible for risk management in medium-sized (50-249 workers) and large companies ( 250 or more workers);

(iii) Elimination of the sample, all participants at any stage of the study, which do not comply with the criteria described above.

The interview consists of two parts:

Part 1 - aimed to realize if the way the estimation develops influences the results of risk assessment and to identify the essential information required for qualitative estimation of risk probability and severity.

Part 2 - intended to identify the required information for qualitative estimation of probability and severity from the perspective of the risk type, by analyzing the observation in real workplace situation (the use of explosives for rock dismantling).

Sixteen face interviews with risk assessment experts were carried out: two were test interviews, other two were excluded because respondents have not confirmed the requirements during the interview and twelve were analyzed (with same number of teachers and risk managers).

In the interview, open questions were used to allow respondents to express their opinion, what they think and know without being influenced by suggestions made by the researcher and avoid the effects of fixed format due to semi-structured model.

\subsection{Processing and analyses of interview data}

To perform the analysis of the interviews, a team of three analysts, all teachers and risk managers in large companies, with the same technical-scientific requirements of the sample of respondents, and which did not participate in any stage of the study was formed. They performed a qualitative analysis of the content of the interviews by applying the complementary techniques of data analysis to avoid the influence of the researcher-interviewer and ensure an independent analysis (triangulation).

The interactive analysis of data with the team of analysts took place in four phases according to the following thematic areas:

(i) Influence of the form of risk estimation in risk assessment;

(ii) Identification of essential information for estimation of probability and severity of occupational risk in general;

(iii) Influence of the type of risk on the information required (markers of information);

(iv) Importance of information available at the time of risk estimation and its influence on risk assessment.

The analysis of interviews was done through the interpretation of the concepts expressed in the con- tents in a qualitative and cognitive approach verifying in:

Phase 1 - the placement of importance-ordered responses per question (1 to 12 );

Phase 2 - the elaboration of individual cognitive maps and respective structure of concepts;

Phase 3 - the construction of the aggregate map, based on the individual structures obtained in the interactions with each analyst, carried out in the previous stage (ordered responses by area and subject);

Phase 4 - the meeting with the three analysts for discussion and looking for a consensus about the final structure of concepts obtained from the group aggregate map according to the purpose of the interview (without researcher intervention).

\subsection{Participants in real workplace situation}

The explosives team composed of six workers: production coordinator, team leader, two fire loaders and two charger fire apprentices.

\section{RESULTS AND DISCUSSION}

\subsection{Results of the qualitative analysis}

In the initial question (Q1) of the interview concerning the form adopted to perform the estimation of probability and severity in a given work situation, and its possible influence on the level of risk (LR), all respondents answered affirmatively and state that the way the technician chooses to proceed with the risk analysis estimation of the two variables have a direct influence on the results of risk assessment (level of risk), when using the SqtRAM.

In the questions relating to the influence of the type of risk (Q3 and Q4), respondents answered largely yes (10 in 12) that the kind of risk influences the information needed to estimate the variables probability and severity in presence of risks related to safety, to occupational hygiene and in high-risk activities. Two respondents reported that the information required is independent of the type of risk analysis, because it should always be gathered according to each situation in the following areas: (i) work organization and procedures; (ii) technical, technological means; (iii) workplace environment; and (iv) human factors.

In the question concerning the influence of the information provided through observation of real work environment (Q5c), workplace case study, all respondents answered affirmatively, that the information available about the workplace is essential to carry out the estimation. Respondents indicated for instance, that the lack of information to estimate the two variables is a factor that leads the technician assigning higher values influencing the results (level of risk) and consequently, the risk control measures proposed. 
Regarding the possible influence of developing risk estimation on the level of risk and on risk assessment results (Q1, Q2b, Q3, Q5c), the answers were always affirmative. The following key points contribute to respondents conviction: the chosen form to estimate (by sector, workplace, function, installation, type of risk); the information gathered prior to the application of the assessment method (tendency to increase value of estimation when the information available is limited) and the type of risk (areas of high risk work, safety and occupational hygiene).

\subsection{Qualitative estimation of probability (markers)}

Several questions were asked to identify markers of information (Q2a, Q5a), understand its influence on the magnitude of risk obtained (Q2b, Q5c) and realize if the type of risk affects markers of information necessary to estimate the probability $(\mathrm{Q} 3)$.

After applying the techniques used for content analysis of the interviews, the need for the following information to estimate probability was identified:

(i) Information on the organization and work processes - the type and sequence of tasks, products used, workload (type and duration of tasks, schedules and breaks), frequency of the task execution or duration of exposure to risk, results of previous risk assessments and controls implemented, outcomes of inspections, compliance status, mobility and rotation of workers and information about accidents (frequency rate);

(ii) Technical, technological and material - adequacy of the technical and technological means to demands, operational conditions of machinery, equipment and tools, existence and standing of fulfillment of maintenance plan (safety manuals) and type of products used;

(iii) Workplace environment - information relating to the physical environment involving the workplace (occupational hygiene and safety conditions);

(iv) Human factors - training, information, experience and the characteristics of each worker with special relevance in behaviors related to safety (individual safety culture).

\subsection{Qualitative estimation of severity (markers)}

Regarding the estimation of the possible consequences caused by the occurrence of exposure to risk, the potential severity of the damage, several questions were raised to identify markers of information needed to estimate this variable (Q2c, Q5b), understand the influence of information available on risk assessment results $(\mathrm{Q} 2 \mathrm{~d}, \mathrm{Q} 5 \mathrm{c})$ and understand if the type of risk affects markers of information required for the qualitative estimation of severity (Q4).

After analysis of the interviews content, the following information considered essential for qualitative estimation of severity was identified: (i) Collective and individual protections are identified as crucial concepts for the estimation;

(ii) Registration and characterization of accidents (severity rate and absenteeism);

(iii) Indications of potential result due to improper use of the means (manuals, safety data sheets);

(iv) Level of emergency planning/training (to reduce damage in the event of an accident).

\subsection{Other information (contributing items)}

Participants also highlighted a set of concepts that are not considered as markers of information for qualitative estimation of the probability and severity, but that should be taken into account as contributing factors and be considered in the risk analysis step, namely: (i) time available to do the risk assessment, (ii) access to the workplaces, (iii) the experience of the evaluator, (iv) methodology and schedule risk assessment, (v) method of estimation, (vi) interaction with employees, (vii) the observation of workload trip and avoid the analysis of each individual task in itself, (viii) unpredictable factors (changes in expected conditions) and (ix) correction of subjectivity in successive risk assessing according to performance indicators and management of OHS.

\subsection{Information available versus variables of semi- quantitative risk assessment methods chosen}

The methods of risk assessment were selected in the semi-quantitative group, based on the following criteria: (i) be considered a semi-quantitative method, (ii) use a simple matrix with two variables or a matrix composed of more than two variables and (iii) be accepted as the most widely used because they are free and available in the literature.

After the identification of the essential information, in order to understand its influence while using semi-quantitative risk assessment methods (mandatory information and technical responsibility) it was performed a juxtaposition of the information identified by the analysts versus the variables of the following chosen methods: (i) simplified method for evaluating accident risks - NTP 330 (National Institute of Safety and Hygiene at Work of Spain), (ii) method of William T. Fine (Cabral \& Veiga 2006) and (iii) the simple matrix method - SMM (Pinto 2008).

The method NTP 330 determines the need for information that makes possible the estimation of the level of exposure - LE (often performing the task and/or exposure time). To estimate the level of disability - LD, information should be gathered on the laws and regulations applicable to the work under analysis to detect compliance / non-compliance through the application of verification techniques in terms of organization and work processes, training, means used and the physical environment of the place. The level of probability - LP, will be found only after gathering the information to estimate the 
level of exposure - LE and the level of disability $\mathrm{LD}$, is the product of the two variables and depends on how they were estimated (LP = LE x LD).

The level of consequence (LC), is the possibility of damage or injury. This method gives more importance to this variable than to the level of probability (LP), as seen through the values of reference established in the scales of valuation, but leaves to the evaluator the responsibility to collect the information considered necessary to estimate the potential damage and reduce subjectivity.

In WT Fine, the second method chosen, the juxtaposition of information is focused only on the degree of hazard, which is the product of three variables $(\mathrm{R}=\mathrm{Fp} \times \mathrm{Fe} \times \mathrm{Fc})$, the exposure factor $(\mathrm{Fe})$, the probability of the accident to occur $(\mathrm{Fp})$ and the consequences if the accident happens $(\mathrm{Fc})$. The risk exposure $(\mathrm{Fe})$ determines that the evaluator should gather information on worker exposure, as he does in method NTP 330 (often performing the task and / or exposure time). To estimate Fp the evaluator is left with the decision of the necessary information, without any of this being determined by the method. The same happens with the variable Fc, which corresponds to the damage / injury possible, once again, it is up to the evaluator to gather the information necessary to estimate the potential damage and thereby reduce the subjectivity of the estimation.

When using the third chosen method, the simple matrix method (SMM), the level of risk is calculated as a function of the independent variables probability (P) and severity (S). This method leaves the decision on the information deemed necessary to estimate the two variables entirely to the evaluator, becoming even more relevant the definition of information considered essential to perform the estimation of risk and the contributing factors presented in the paragraph "other information" (e.g. experience of the evaluator and the methodology used), to estimate realistically the two variables.

\section{FINAL REMARKS}

\subsection{Results of research hypotheses}

Regarding the derivative questions raised in this study (DQ1 and DQ2), the validation of the hypotheses points to the importance of the available information in qualitative risk analysis, taking into consideration that:

(i) There are significant differences in the level of risk when we use different information (amount and type of information available) to estimate the variables probability and severity of accidents. Lack of information leads the evaluator in technical terms to assign higher values in accordance with the reference scales, also when the type of information is inadequate leads to a higher subjectivity of risk as- sessment and makes it difficult to fix the deviations in successive evaluations;

(ii) The type of risk influences the choice of information needed to estimate these two variables. The criteria to gather information must meet the risk typology of the activities and workplace analyzed, in accordance with work safety, occupational hygiene and high risk activities contexts.

\subsection{Contributions to the risk assessment}

In this work information considered essential was identified for the qualitative estimation of risk when semi-quantitative risk assessment methods are used, concerning the organization and work procedures, technical and technological means employed, physical environment and human factors which characterize the working conditions.

In the qualitative analysis of interview contents, a set of concepts identified as contributing factors were also raised and should be taken into account by the person responsible for risk assessment.

The information identified substantiates the descriptors of the chosen methods, the technical responsibility and the reduction of subjectivity in the estimation of risk.

\section{REFERENCES}

Cabral, F. \& Veiga, R. 2006. Higiene, segurança e saúde e prevenção de AT (vol. 1, 20ª ed.). Lisboa: Verlag Dashöfer.

Eden, C. \& Ackermann, F. 1998. Making strategy: The journey of strategic management. London: SAGE Publications.

Freitas, L.C. 2008. Manual de SST. Lisboa: Edições Sílabo.

Fortin, M. 2006. O processo de investigação: Da conceção à realização ( $3^{\mathrm{a}}$ ed.). Loures: Lusociência.

Lluna, G. 2003. Sistema de gestión de riesgos laborales e industriales. Madrid: Edições Fundación Mapfre.

Northcutt, N. \& McCoy, D. 2004. Interactive qualitative analysis: A systems method for qualitative research. Thousand Oaks, CA: SAGE Publications.

Nunes, F. 2010. Segurança e saúde no trabalho: Manual técnico (3 ${ }^{\mathrm{a}}$. ed.). Amadora: Edições Gustave Eiffel.

Pinto, P. 2008. Manual segurança: Construção, conservação e restauro de edifícios ( $3^{\mathrm{a}}$. ed.). Lisboa: Edições Sílabo.

Romero, J.C. 2004. Métodos de evaluación de riesgos laborales. Madrid: Ediciones Diaz de Santos. 